\title{
Amniotic Membrane Transplantation as a Treatment for Sterile Infiltration and Corneal Melting after Corneal Crosslinking for Keratoconus
}

\author{
Takanori Sasaki $^{a} \quad$ Takeshi Ide $^{b}$ Ikuko Toda ${ }^{c}$ Naoko Kato ${ }^{a}$ \\ ${ }^{a}$ Department of Ophthalmology, Saitama Medical University, Iruma, Japan; ${ }^{\text {b}}$ Tokyo Vision \\ Eye Clinic Asagaya, Tokyo, Japan; ' Minamiaoyama Eye Clinic, Tokyo, Japan
}

\section{Keywords}

Amniotic membrane transplantation · Sterile infiltration · Corneal melting · Corneal crosslinking $\cdot$ Keratoconus

\begin{abstract}
We report a case of corneal melting through sterile infiltration presumably due to excessive use of nonsteroidal anti-inflammatory eye drops after corneal crosslinking (CXL). It was treated using steroids combined with amniotic membrane transplantation (AMT). A 33-year-old man with progressing keratoconus underwent left eye CXL. We prescribed betamethasone and levofloxacin eye drops 5 times daily and diclofenac sodium eye drops 3 times daily for 3 days. Three days after $C X L$, there was a persistent epithelial defect and the left corneal stroma was clouded. His visual acuity remained unchanged. We prescribed betamethasone hourly and $20 \mathrm{mg}$ prednisolone daily. At 1 week after $\mathrm{CXL}$, the corneal epithelial defect and infiltration were unchanged. He had not stopped using diclofenac at the prescribed time. On day 17, we performed AMT and his cornea and visual acuity improved. AMT may be effective against persistent epithelial defects and corneal melting after CXL.




\section{Introduction}

Corneal collagen crosslinking (CXL) was first reported by Wollensak et al. in 2003 [1], using riboflavin as a photosensitizer being excited by ultraviolet A light (UVA) exposure. CXL is known to halt the progression of keratoconus. Although CXL has been considered a relatively safe treatment, a few complications such as sterile infiltrates (7.6\%) and stromal scars (2.8\%) have been reported [2].

Nonsteroidal anti-inflammatory drugs (NSAIDs) are used for control of acute or chronic pain or inflammation. NSAIDs inhibit cyclooxygenases and decrease prostaglandin production; their use for corneal wound healing is a matter of debate. Postoperative use of topical NSAIDs promotes faster wound healing [3]; however, Lee et al. [4] investigated the toxicity of NSAID eye solutions against corneal epithelial cells in vitro. Asai et al. [5] reported 3 cases of corneal melting caused by bromfenac sodium used to treat surface and postoperative inflammation.

Moreover, Gokhale and Vemuganti [6] reported a case of corneal melting and perforation 1 week after CXL for keratoconus. They speculated the concomitant postoperative use of topical diclofenac and proparacaine eye drops after CXL as the predisposing risk factors. Mohamed-Noriega et al. [7] presented a case of corneal melting and perforation 6 weeks after CXL for keratoconus. They proposed postoperative nepafenac eye drop use, a cornea thinner than $400 \mu \mathrm{m}$, and diabetes mellitus as the predisposing risk factors.

We report a case of corneal melting through sterile infiltration presumably due to excessive use of NSAID eye drops, treated with topical and general steroid applications, which finally required amniotic membrane transplantation (AMT).

\section{Case Report}

A 33-year-old man visited our institute complaining of vision reduction in his left eye. He had no significant personal or family history. His visual acuity was $0.2(0.9 \times \mathrm{s}-1.00 \mathrm{D} \mathrm{c}$ $-2.00 \mathrm{D} \mathrm{AX} 65^{\circ}$ ), OS. Slit lamp examination showed a cone-shaped cornea and corneal thinning. Intraocular pressure was $11 \mathrm{~mm} \mathrm{Hg}$. Anterior segment optical coherence topography (CASIA ${ }^{\circledR}$; Tomey, Aichi, Japan) indicated that the steepest keratometric value was $48.6 \mathrm{D}$, and the thinnest corneal thickness was $441 \mu \mathrm{m}$, OS. He was diagnosed with keratoconus in the left eye. After one and a half year, his visual acuity deteriorated to $0.3(0.7 \times \mathrm{s}-1.50 \mathrm{D}$ c -3.00 $\left.\mathrm{D} A X 5^{\circ}\right), \mathrm{OS}$, and the steepest keratometric value increased to $49.7 \mathrm{D}$; CXL on his left eye was planned. His thinnest corneal thickness was $445 \mu \mathrm{m}$, OS. After topical anesthesia, the central epithelium of his left cornea was debrided with $20 \%$ ethanol. An accelerated protocol with $0.1 \%$ riboflavin instillation for 20 min followed by UVA irradiation with $18.0 \mathrm{~mW} / \mathrm{cm}^{2}$ for 5 min (KXL System ${ }^{\circledR}$; Avedro, Waltham, MA, USA) was performed. After UVA irradiation, a bandage contact lens was placed over the cornea following our standard protocol. Postoperatively, betamethasone and levofloxacin eye drops 5 times daily and diclofenac sodium eye drops 3 times daily were prescribed. The patient was instructed to stop the use of the diclofenac sodium eye drops after 3 days.

On the day after CXL, his left cornea revealed a corneal epithelial defect and mild ciliary injection, but the underneath stroma was translucent, and no remarkable infiltration was observed. Three days after CXL, his left cornea revealed a persistent epithelial defect, and the underneath corneal stroma was clouded. Severe ciliary injection was also observed. His visual acuity was 0.01 (noncorrigunt), OS. We estimated that the corneal lesion was not an in- 
Case Reports in
Ophthalmology

Case Rep Ophthalmol 2018;9:185-189

DOI: $10.1159 / 000487074$

C 2018 The Author(s). Published by S. Karger AG, Basel www.karger.com/cop

Sasaki et al.: Amniotic Membrane Transplantation as a Treatment for Sterile Infiltration and Corneal Melting after Corneal Crosslinking for Keratoconus

fection from the clinical feature. We scraped the surface of corneal erosion and carried out a bacterial examination on day 3 , but no microbial agent was detected. We instructed the patient to use betamethasone eye drops every hour and also prescribed $20 \mathrm{mg}$ of oral prednisolone per day. One week after CXL, the corneal epithelial defect and infiltration were unchanged. At the follow-up visit, he reported that he had continued the use of diclofenac sodium eye drops 5 times per day for 5 days, despite our presurgical instructions. We prescribed $20 \%$ serum eye drops 5 times per day. Nevertheless, that area did not show epithelialization; superficial corneal stroma melt was observed, and the underneath stroma was visibly edematous (Fig. 1).

Twelve days after CXL, we performed AMT on his left cornea, and we covered the entire corneal surface with an amniotic membrane, sutured it with 8-0 vicryl. After that, his cornea showed improvement. The amniotic membrane was dissolved about 2 weeks after AMT. The epithelium began to recover thereafter, and the underneath stroma became dehydrated (Fig. 2). We removed the bandage contact lens 50 days after AMT. His visual acuity had improved to $0.2\left(0.7 \times \mathrm{s}+0.25 \mathrm{D} \mathrm{c}-3.50 \mathrm{D}, \mathrm{AX} 100^{\circ}\right)$, OS 60 days after CXL (48 days after AMT), and we stopped the use of the serum eye drops.

\section{Discussion}

In the present case, persistent epithelial defect, sterile infiltration, and corneal melting were probably caused by excessive postoperative use of NSAIDs. The corneal involvement could not be improved by intensive topical and general steroid application; however, it finally improved after performance of AMT.

In the present case, the excessive use of topical NSAIDs by the patient, despite our presurgical instructions, presumably caused delayed re-epithelialization, and proinflammatory cytokines released from the damaged epithelial cells further obscured epithelial healing. NSAIDs are widely used after various ocular surgeries, expecting anti-inflammatory and analgesic effects via inhibition of the enzymes cyclooxygenase $1 / 2$. On the other hand, the toxicity of NSAIDs against corneal re-epithelialization has been investigated both in vitro [4] and in vivo [8]. Çerman et al. [9] suggested that proinflammatory cytokines directly secreted from damaged epithelial cells may be released into the stroma and consequently trigger the migration of inflammatory cells to the affected stroma.

For the treatment against sterile infiltration and corneal melting, topical and general steroid application has been reported to be effective [10]. However, steroid application had only limited effect in the present case. Therefore, we performed AMT on the 17th day after CXL. AMT, which was first used by Kim and Tseng [11] for ocular surface reconstruction, has recently found widespread use in ophthalmology as a patch for conjunctival reconstruction [12], corneal surface reconstruction [12], forniceal reconstruction [12], and glaucoma surgery [13]. The amniotic membrane has been reported to contain growth factors that promote epithelial wound healing on the ocular surface [14]. Anti-inflammatory and antiscarring effects of the amniotic membrane have been reported [15]. Moreover, in the present case, AMT reduced inflammation, promoted epithelial healing, and consequently halted corneal melting. Although corneal stromal opacity remained, visual acuity recovered, and the corneal curvature returned to its preoperative condition.

The present case may indicate the possible risk of NSAIDs after CXL regarding epithelial healing. After encountering the present case, we no longer prescribe diclofenac sodium eye drops postoperatively. Frequent observation within 1 week after CXL is crucial. Further- 
Sasaki et al.: Amniotic Membrane Transplantation as a Treatment for Sterile Infiltration and Corneal Melting after Corneal Crosslinking for Keratoconus

more, one should consider that AMT could be effective in cases where persistent epithelial defects and corneal melting occur.

\section{Statement of Ethics}

The authors have no ethical conflicts to disclose.

\section{Disclosure Statement}

The authors have no conflicts of interest to disclose.

\section{References}

1 Wollensak G, Spoerl E, Seiler T: Riboflavin/ultraviolet-A-induced collagen crosslinking for the treatment of keratoconus. Am J Ophthalmol 2003;135:620-627.

2 Koller T, Mrochen M, Seiler T: Complication and failure rates after corneal crosslinking. J Cataract Refract Surg 2009;35:1358-1362.

3 Barba KR, Samy A, Lai C, Perlman JI, Bouchard CS: Effect of topical anti-inflammatory drugs on corneal and limbal wound healing. J Cataract Refract Surg 2000;26:893-897.

-4 Lee JS, Kim YH, Park YM: The toxicity of nonsteroidal anti-inflammatory eye drops against human corneal epithelial cells in vitro. J Korean Med Sci 2015;30:1856-1864.

5 Asai T, Nakagami T, Mochizuki M, Hata N, Tsuchiya T, Hotta Y: Three cases of corneal melting after instillation of a new nonsteroidal anti-inflammatory drug. Cornea 2006;25:224-227.

Gokhale NS, Vemuganti GK: Diclofenac-induced acute corneal melt after collagen crosslinking for keratoconus. Cornea 2010;29:117-119.

7 Mohamed-Noriega K, Burtrón-Valdez K, Vazquez-Galvan J, Mohamed-Noriega J, Cavazos-Adame H Mohamed-Hamsho J: Corneal melting after collagen cross-linking for keratoconus in a thin cornea of a diabetic patient treated with topical nepafenac: a case report with a literature review. Case Rep Ophthalmol 2016;7:119-124.

8 Hersh P, Rice B, Baer J, Wells PA, Lynch SE, McGuigan LJ, Foster CS: Topical nonsteroidal agents and corneal wound healing. Arch Ophthalmol 1990;108:577-583.

-9 Çerman E, Özcan DÖ, Toker E: Sterile infiltrates after corneal collagen cross-linking: evaluation of risk factors. Acta Ophthalmol 2017;95:199-204.

10 Sanchez J, Polack FM: Effect of topical steroids on the healing of corneal endothelium. Invest Ophthalmol 1974;13:17-22.

$\checkmark 11$ Kim JC, Tseng SC: Transplantation of preserved human amniotic membrane for surface reconstruction in severely damaged rabbit corneas. Cornea 1995;14:473-484.

12 Tseng SC, Prabhasawat P, Lee SH: Amniotic membrane transplantation for conjunctival surface reconstruction. Am J Ophthalmol 1997;124:765-774.

13 Fujishima H, Shimazaki J, Shinozaki N, Tsubota K: Trabeculectomy with the use of amniotic membrane for uncontrollable glaucoma. Ophthalmic Surg Lasers 1998;29:428-431.

$\checkmark 14$ Koizumi N, Inatomi T, Sotozono C, Fullwood NJ, Quantock AJ, Kinoshita S: Growth factor mRNA and protein in preserved human amniotic membrane. Curr Eye Res 2000;20:173-177.

15 Tseng SC, Espana E, Kawakita T, Di Pascuale MA, Li W, He H, Liu T, Cho T, Gao Y, Yeh L, Liu CY: How does amniotic membrane work? Ocul Surf 2004;2:177-187. 


\section{Case Reports in Ophthalmology}

\begin{tabular}{l|l}
\hline Case Rep Ophthalmol 2018;9:185-189 \\
\hline DOI: 10.1159/000487074 & $\begin{array}{l}\text { @ 2018 The Author(s). Published by S. Karger AG, Basel } \\
\text { www.karger.com/cop }\end{array}$ \\
\hline
\end{tabular}

Sasaki et al: Amniotic Membrane Transplantation as a Treatment for Sterile Infiltration and Corneal Melting after Corneal Crosslinking for Keratoconus
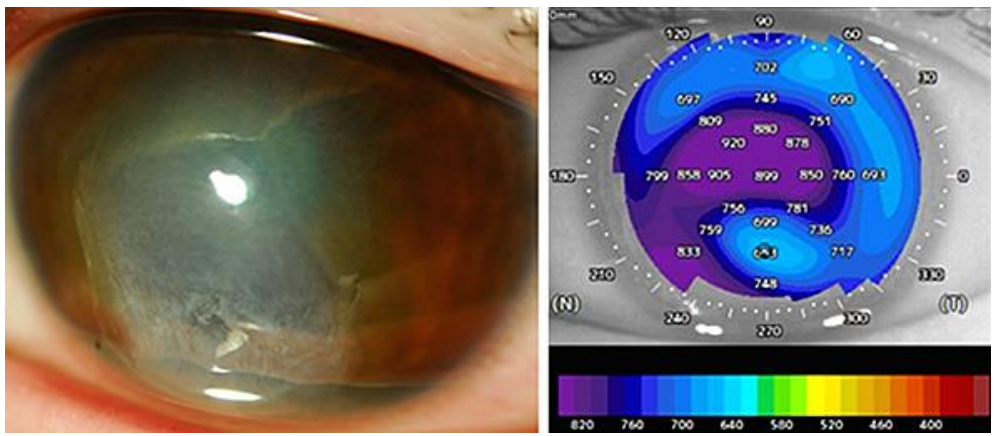

Fig. 1. Clinical photograph and corneal pachymetry map 3 days after corneal crosslinking. Left: Slit-lamp microscopy shows that the persistent epithelial defect remains. The underneath corneal stroma is melted and clouded by edema. Right: Pachymetry map shows the corneal swelling.
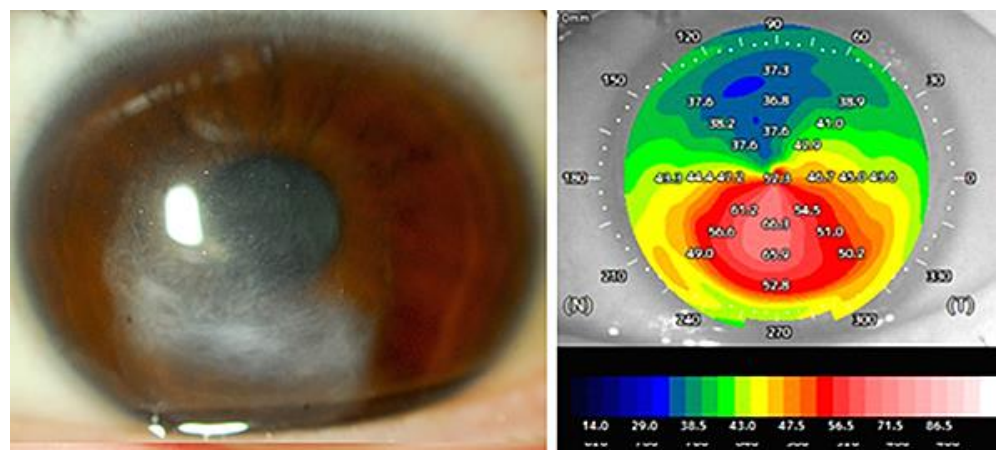

Fig. 2. Clinical photograph and corneal pachymetry map 3 months after crosslinking. Left: Epithelialization is achieved and the corneal stroma shows mild scarring. Right: The axial map of the left cornea shows that the steepest keratometric value improved to 54.0 D after crosslinking. 